\title{
Aprendizado de Geometria Analítica e Álgebra Linear Utilizando um Software Gráfico via Internet
}

\author{
Igor R. Lima ${ }^{1}$, Maria do Carmo P. de T. Costa ${ }^{2}$, Heitor A. X. Costa ${ }^{3}$ \\ ${ }^{1}$ Grupo de Pesquisa em Engenharia de Software (PqES) - Departamento da Ciência da \\ Computação - Universidade Federal de Lavras (UFLA) \\ Caixa Postal 3037 - CEP 37200-000 - Lavras - MG - Brazil \\ ${ }^{2}$ Departamento de Ciências Exatas - Universidade Federal de Lavras (UFLA) \\ Caixa Postal 3037 - CEP 37200-000 - Lavras - MG - Brazil \\ ${ }^{3}$ Grupo de Pesquisa em Engenharia de Software (PqES) - Departamento da Ciência da \\ Computação - Universidade Federal de Lavras (UFLA) \\ Caixa Postal 3037 - CEP 37200-000 - Lavras - MG - Brazil \\ 1igorlima@comp.ufla.br, ${ }^{2}$ mctoledo@ufla.br, ${ }^{3}$ heitor@ufla.br
}

\begin{abstract}
The computational tools, including educational software and hypertext documents, allow the teaching of mathematics so innovative, strengthening the role of graphic language. Therefore, it is another way to seek and facilitate the construction of knowledge, more autonomous and independent, and, by using the computer, especially with exploratory tools; some approximation of concrete materials is possible. This work proposes the TBC-GAAL/WEB, educational software, to be used in disciplines of Analytical Geometry and Linear Algebra; as possible use it through the Web, its spread is high, not being restricted to the site of their development.
\end{abstract}

Key words: Informatics in Education, Educational tools.

Resumo: As ferramentas computacionais, incluindo produtos de software educacionais e documentos hipertexto, permitem o ensino da matemática de modo inovador, reforçando o papel da linguagem gráfica. Assim, é uma outra maneira de buscar e viabilizar a construção do conhecimento, de forma mais autônoma e independente e, ao utilizar o computador, principalmente com ferramentas exploratórias, uma certa aproximação dos materiais concretos é possível. Esse trabalho propõe um software educacional, TBC-GAAL/WEB (Treinamento Baseado em Computador para Geometria Analítica e Álgebra Linear via Web) a ser usado em disciplinas de Geometria Analítica e Álgebra Linear; por ser possível utilizá-lo via Web, a sua difusão é alta, não ficando restrito ao local de seu desenvolvimento.

Palavras-chave: Informática na Educação, Ferramentas Educacionais.

\section{Introdução}

As primeiras iniciativas para o uso de computadores na educação brasileira tiveram suas raízes na década de setenta, quando em 1971, pela primeira vez, discutiu-se o uso de computadores no ensino de Física. Em 1981, ocorreu o I Seminário Nacional de Informática na Educação, que foi responsável por destacar a importância de pesquisar o uso do computador como ferramenta auxiliar do processo de ensino-aprendizagem. 
Em particular, com relação à Matemática, as ferramentas computacionais (p. e. software educacional) permitem o seu ensino de modo inovador, reforçando o papel da linguagem gráfica e relativizando a importância do cálculo. Segundo Ponte (2006), elas constituem um meio educacional auxiliar para apoiar a aprendizagem dos alunos e permitem criar situações de aprendizagem estimulante. De acordo com Barufi (2006), é uma outra maneira de buscar e viabilizar a construção do conhecimento, de maneira mais autônoma e independente, em um novo ambiente, onde os movimentos e as interações são diferentes e obedecem a novos modelos. Além disso, abrem um novo leque de possibilidades em função das inúmeras simulações que podem ser realizadas e dos questionamentos que podem e precisam ser estabelecidos. Ainda segundo Barufi (2006), ao usar o computador, principalmente com ferramentas exploratórias, é possível uma certa aproximação dos materiais concretos, ajudando os estudantes na construção de raciocínios formais.

Esse trabalho vai ao encontro dessa aproximação, propondo um software educacional (TBC-GAAL/WEB) a ser usado em disciplinas de Geometria Analítica e Álgebra Linear. O TBC-GAAL/WEB cria novas e promissoras perspectivas de expansão dos limites da sala de aula, permitindo aos alunos uma forma independente de construir seu conhecimento matemático. Ele permite aliar teoria e prática e uma abordagem interdisciplinar e contribui tecnologicamente com a sociedade.

O artigo está organizado da seguinte forma: a seção 2 apresenta a importância do uso do computador na educação; a seção 3 detalha algumas ferramentas para o ensino de Geometria Analítica e Álgebra Linear; a seção 4 apresenta o TBC-GAAL/WEB; e a seção 5 apresenta algumas conclusões e sugestões de trabalhos futuros.

\section{A Importância do Uso do Computador na Educação}

O papel da Informática como ferramenta educativa tem crescido de forma significativa nos últimos anos e a Informática na Educação é, hoje, uma das áreas mais fortes da Tecnologia Educacional [Cysneiro, 2006].

Fazendo uso de uma ferramenta computacional, o ato de aprender pode se tornar mais simples e prazeroso; o estudante motivado pode aprimorar o seu conhecimento e o interesse em aprender é despertado no aluno com o uso de ambientes lúdicos que harmonizam o conhecimento e a prática dos conceitos repassados em sala de aula.

O uso de um software é importante como facilitador da aprendizagem, uma vez que possibilita ao estudante utilizar uma ferramenta flexível e adaptável ao perfil individual de cada um e desenvolver sua própria criatividade.

Não se pode deixar de destacar que, ao interagir com um software educacional, os estudantes são encorajados para troca de experiências, viabilizando o processo de aprendizagem em grupo e permite a construção do conhecimento de forma mais autônoma e independente, ao utilizar um universo que possibilita interações, não necessariamente presenciais.

Segundo Santos e Costa (2006), a elaboração de uma ferramenta computacional para fins didáticos deve tornar o ensino do conteúdo abordado mais prático, de forma a despertar o interesse do aluno, o seu espírito de pesquisa e a busca de informações que possam torná-lo um profissional crítico e de opinião sólida. Usualmente, um estudante se interessa por aulas dinâmicas, criativas e não tradicionais, pois prende sua atenção e reflete no seu desempenho de maneira positiva. 


\section{Ambientes Computacionais Educacionais para o Ensino de Geometria Analítica e Álgebra Linear}

Nesta seção, são citados alguns projetos relacionados. Deve-se ressaltar, contudo, que não houve pretensão de fazer uma análise exaustiva de tais projetos. São eles:

- WinPlot: programa gráfico de propósito geral, desenvolvido pelo Professor Richard Parris da Philips Exeter Academy. Seu uso no âmbito da geometria plana e espacial é interessante, consistindo de uma ferramenta didática, uma vez que o WinPlot permite traçar e animar gráficos em $2 \mathrm{D}$ e 3D, por meio de vários tipos de equações (explícitas, implícitas, paramétricas, entre outras). O WinPlot foi desenvolvido na linguagem de programação C++ [Parris (a), 2008];

- C.a.R (Compasses and Ruler): foi desenvolvido pelo Professor René Grothman. Este software possui ferramentas para construções geométricas planas com o uso de régua e de compassos. Por exemplo, com o uso do mouse, são possíveis marcar pontos na tela, transportar distâncias, traçar retas e circunferências e traçar paralelas e perpendiculares. Os diagramas típicos encontrados em texto de geometria plana podem ser feitos com rapidez e precisão usando o mouse. No entanto, ao contrário do mundo real, as construções geométricas virtuais feitas no C.a.R. não ficam eternamente estáticas; elas se mexem sob um comando. Para ser mais preciso, as relações matemáticas, que vigoram entre a construção dos pontos geométricos iniciais e os demais objetos, não são destruídas ao movimentar o mouse. A sua principal característica é poder estudar uma mesma construção para diferentes configurações de pontos sem a necessidade de repetir a construção. O C.a.R. foi desenvolvido na linguagem de programação Java [Grothman, 2008];

- Yacas (yet another computer algebra system); é um software para álgebra computacional. A linguagem possui variáveis e funções definidas pelo usuário. Há uma implementação básica para polinômios de uma variável, integração de funções e cálculo de tensores. Além disso, entre as funções implementadas, há: i) aritmética racional; ii) computação vetorial, complexa e matricial (dentre essas, cálculo de inversas e determinantes e a solução de sistemas de equações); iii) derivadas; iv) previsão arbitrário; v) séries de Taylor; e vi) soluções numéricas (métodos de Newton). O Yacas foi desenvolvido na linguagem de programação Java [Pinkus, 2008];

- WinLab: foi desenvolvido pelo Professor Richard Parris e inclui sete sub programas: i) seções cônicas; ii) polígonos da estrela; iii) um utilitário para encontrar raízes de funções elementares; iv) visualização 2D; v) gráficos funcionais aleatórios para que o usuário possa identificar; vi) realiza rotações de curvas; e vii) realiza translações de curvas. Os dois últimos itens utilizam as equações do tipo $\mathrm{ax}^{2}+\mathrm{bxy}+\mathrm{cy}^{2}+\mathrm{dx}+$ ey $+\mathrm{f}=0$, informando a nova equação da curva. $\mathrm{O}$ WinLab foi desenvolvido na linguagem de programação C++ [Parris (b), 2008];

- Wingeom: foi desenvolvido pelo Professor Richard Parris, sendo de fácil uso para o usuário, é de domínio público e possui várias ferramentas que possibilitam a realização de atividades interessantes e dinâmicas, dentre as quais, podem-se destacar as construções geométricas em duas e três dimensões e, por meio de animação, é possível verificar diversas propriedades geométricas. Nota-se uma certa instabilidade, pois o Wingeom está em constante desenvolvimento, devido às diversas alterações que são freqüentemente feitas conforme as necessidades dos usuários. Há versões em português e em várias outras línguas. O Wingeon foi desenvolvido na linguagem de programação C++ [Parris (c), 2008]. 


\section{TBC-GAAL/WEB}

\subsection{Tecnologia Utilizada}

O TBC-GAAL foi desenvolvido na linguagem de programação Java por utilizar um paradigma da programação orientado a objetos e, também, pela facilidade que esta linguagem proporciona para a criação de software interativo em um mundo em que a Internet e a World Wide Web têm suma importância [Deitel, 2006].

A linguagem de programação Java foi baseada nas duas linguagens de implementação mais amplamente difundidas no mundo, $\mathrm{C}$ e C++. Essa nova linguagem tem removido os recursos mais confusos, mais complexos e mais propensos a erro de $\mathrm{C} / \mathrm{C}++$ (como sobrecarga de operadores e herança múltipla, entre outros). Isso possibilitou uma linguagem concisa, sem os recursos de uso especial que eram utilizados apenas por pequenos segmentos da comunidade de programação. Além disso, torna os programas desenvolvidos com esta linguagem de programação portáveis, fazendo que esta seja apropriada para a implementação de aplicativos baseados na Internet e na World Wide Web. Inclui os recursos de que o projeto TBC-GAAL realmente precisa como: imagens gráficas, componentes de interface gráfica com o usuário, tratamento de exceções, multithreading, multimídia e estruturas de dados préempacotadas.

O TBC-GAAL foi desenvolvido com código orientado a objeto e baseado em applets, os quais são programas Java que podem ser embutidos em documentos Hypertext Markup Language. Assim, quando um navegador carrega a página Web que contém o applet, ele é baixado para o navegador e começa a ser executado.

\subsection{Uso do Softwre}

Uma das características interessantes que o TBC-GAAL/WEB possui é o seu fácil acesso às pessoas que tenham um computador conectado à rede mundial de computadores com a máquina virtual Java instalada. Com isso, a sua difusão é alta, não ficando o seu uso restrito ao local de seu desenvolvimento.

O TBC-GAAL/WEB é uma ferramenta valiosa para a formação de alunos, pois atende as disciplinas que estão no início do curso e que são pré-requisitos necessários para conteúdos mais específicos de períodos avançados. Quando feita uma boa base, o rendimento e o desempenho aumentam, proporcionando melhores resultados, melhores currículos e melhores profissionais para o mercado.

O TBC-GAAL/WEB apresenta uma tela principal contendo uma barra de menu com os temas: i) Vetores, há opções para o aprendizado de operações com vetores; ii) Cônicas, há as opções para o aprendizado de cônicas que contém um recurso animado para visualizar as definições; e iii) Quádricas, opções para a representação gráfica das quádricas.

A Figura 1 apresenta o submenu Vetores, onde mostra quais operações podem ser feitas entre vetores. A opção de Sistema de Coordenadas 3D mostra ao usuário como representar um ponto no espaço.

Ao escolher a opção Vetores $\rightarrow$ Sistemas de Coordenadas 3D, é apresentada uma tela que permite ao usuário preencher três campos com os valores das coordenadas $\mathrm{x}, \mathrm{y}$ e z. Após pressionar o botão $\mathrm{OK}$, duas representações destes pontos no espaço são apresentadas, sendo uma em forma de cubo e a outra por retas paralelas aos eixos, de modo a facilitar a visualização (Figura 2). 


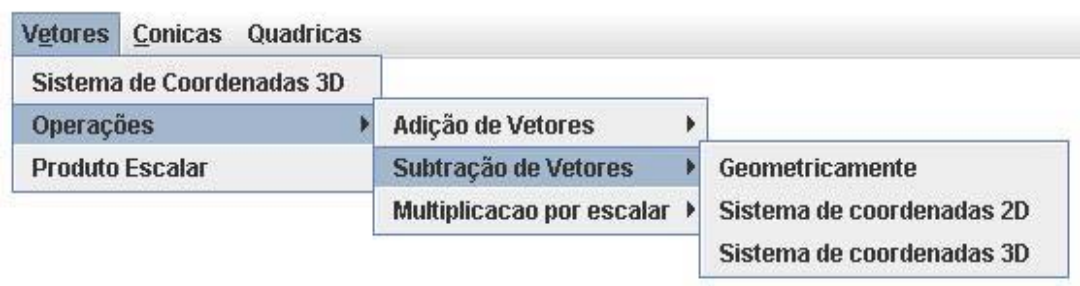

Figura 1 - Submenu do Tema Vetores

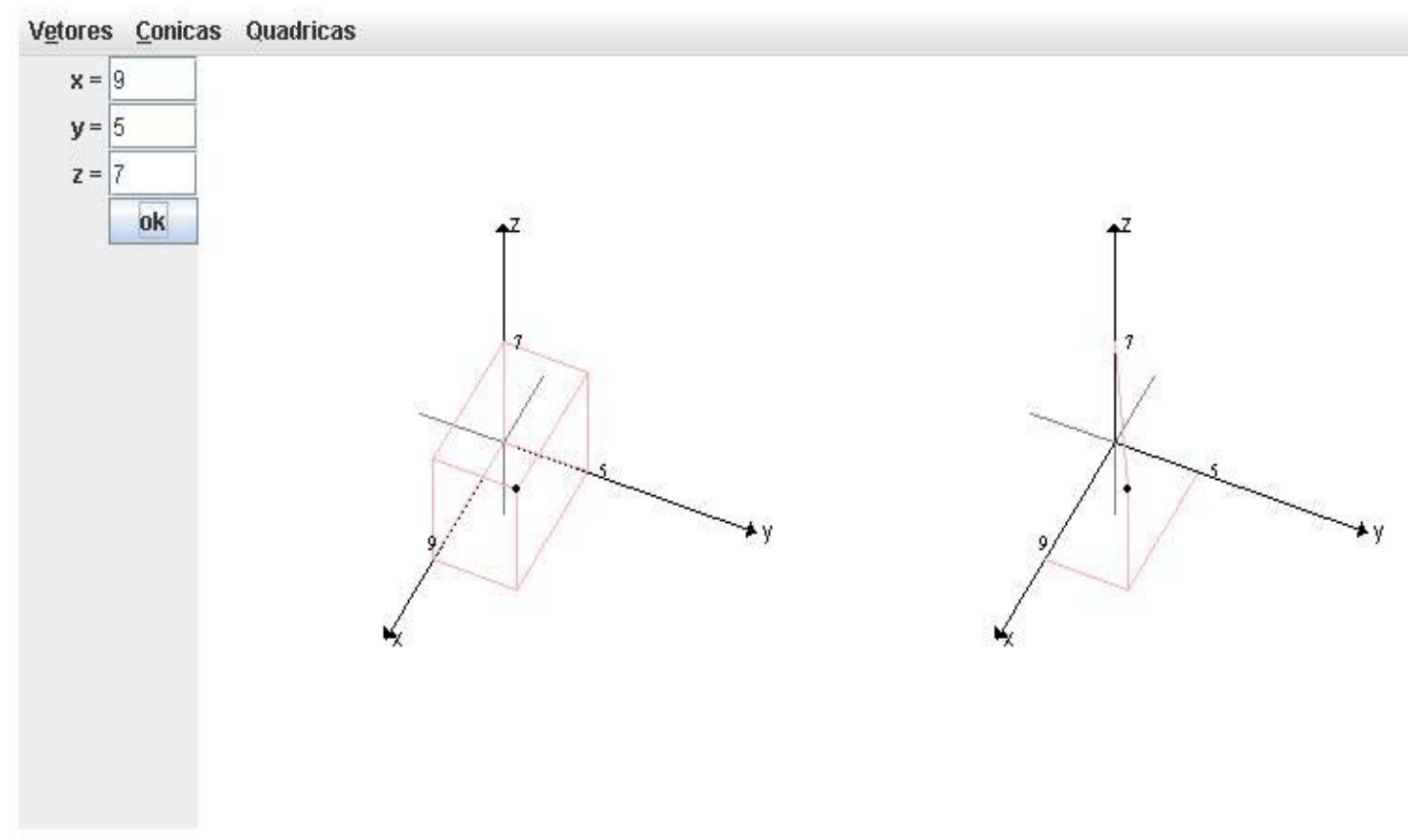

Figura 2 - Sistema de coordenadas, formado por três retas perpendiculares x, y e z.

A Figura 3 mostra a soma de dois vetores. O usuário desenha os vetores, clicando e arrastando o mouse, e escolhe a regra que deseja usar (Regra do Paralelogramo ou Fechar o Triângulo). Após ativar a opção desejada, os vetores movem-se até que fiquem posicionados adequadamente para que a soma seja feita conforme a regra escolhida.

A Figura 4 apresenta a soma de dois vetores no espaço. Nesta figura, existem 6 campos que devem ser preenchidos com os valores das coordenadas dos vetores. A tela 
é dividida em duas, uma com os vetores a serem somados e a outra com o vetor Soma. Essa divisão possibilita uma melhor visualização.

Vetores Conicas Quadricas

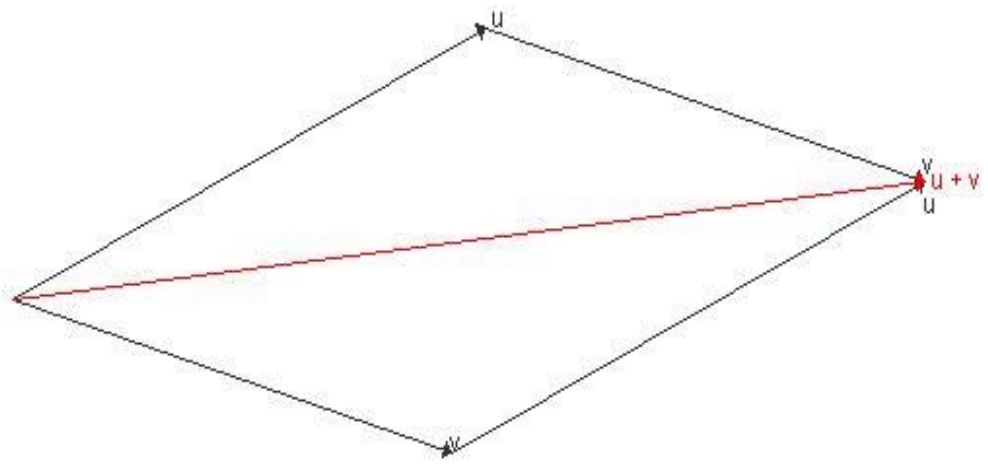

Regra do Paralelogramo

Fechar 0 Triangulo

Limpar Painel

Figura 3 - Tela para Apresentar a soma de dois vetores (Regra do Paralelogramo)

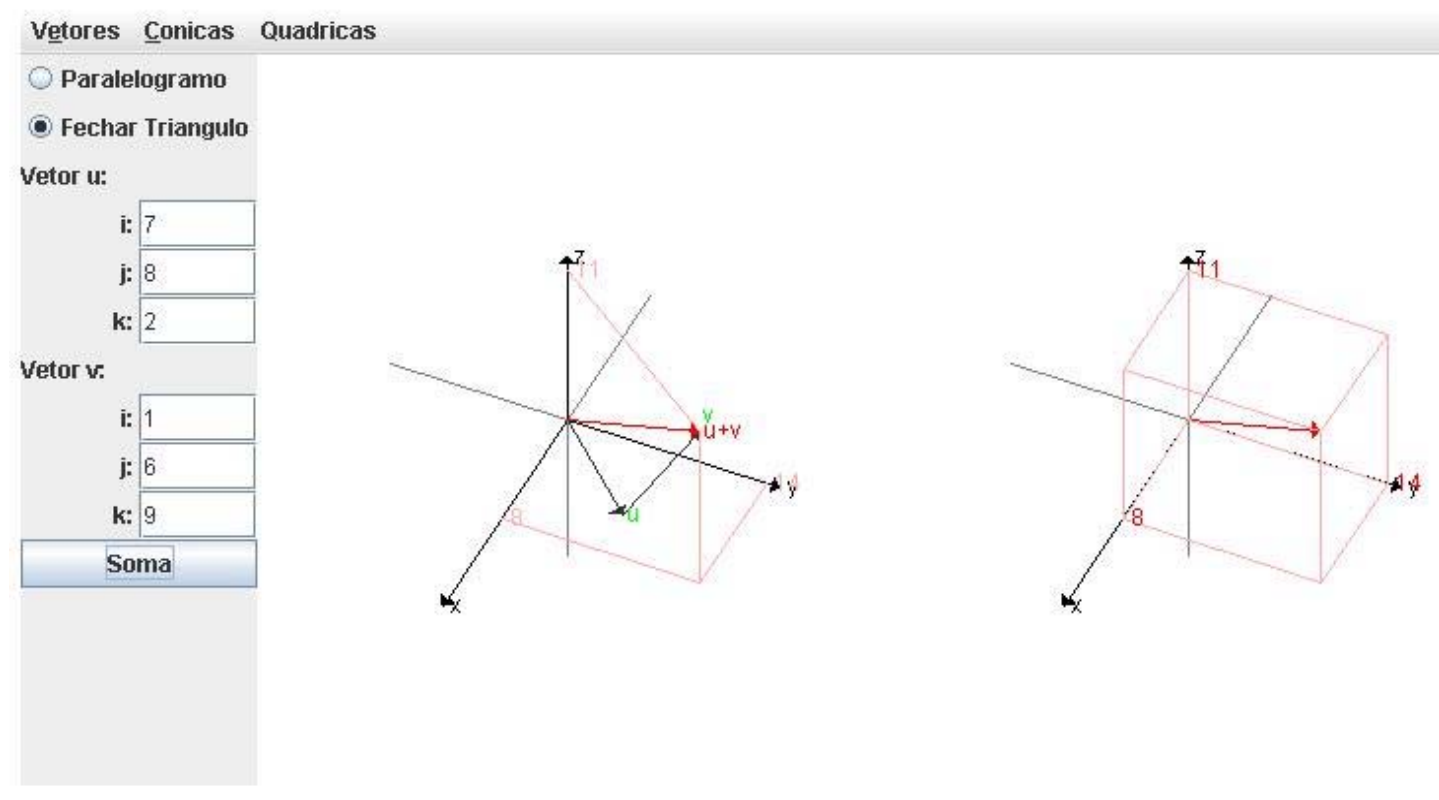

Figura 4 - Tela para Definir e Apresentar a Soma de Dois Vetores no Espaço

Após preencher os campos das coordenadas dos vetores e ativar a operação Produto Escalar, os valores das normas e da projeção são mostrados na tela, além de mostrar graficamente (Figura 5).

A Figura 6 apresenta a construção de uma elipse, utilizando uma animação que mostra o seu traçado, simulando o uso de um laço completo de barbante e dois pregos. Os pregos são fixados em dois pontos (chamados focos) e é deslizado um lápis sobre o 
papel de forma que, apoiando-se nos pregos e na ponta do lápis, o laço de barbante se mantenha esticado.
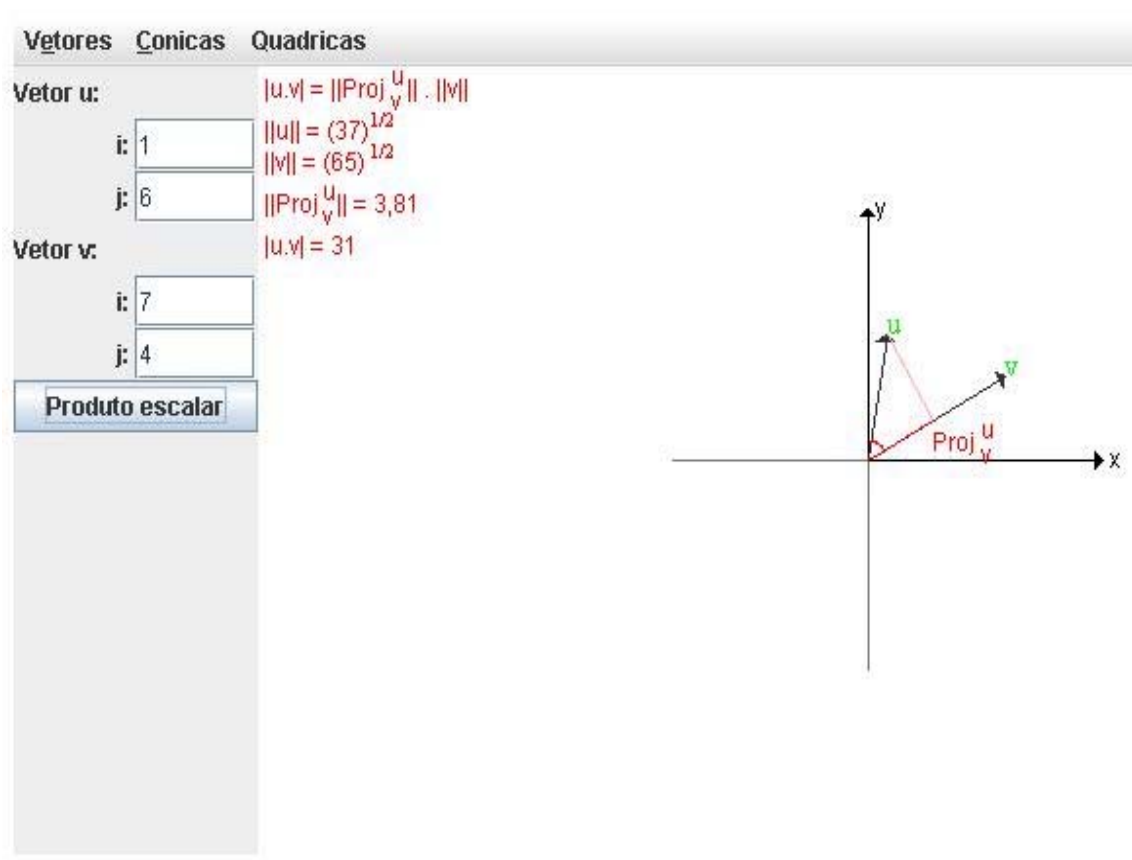

Figura 5 - Definir e Apresentar o Produto Escalar dos vetores $u=(x 1, y 1)$ e $v=(x 2, y 2)$

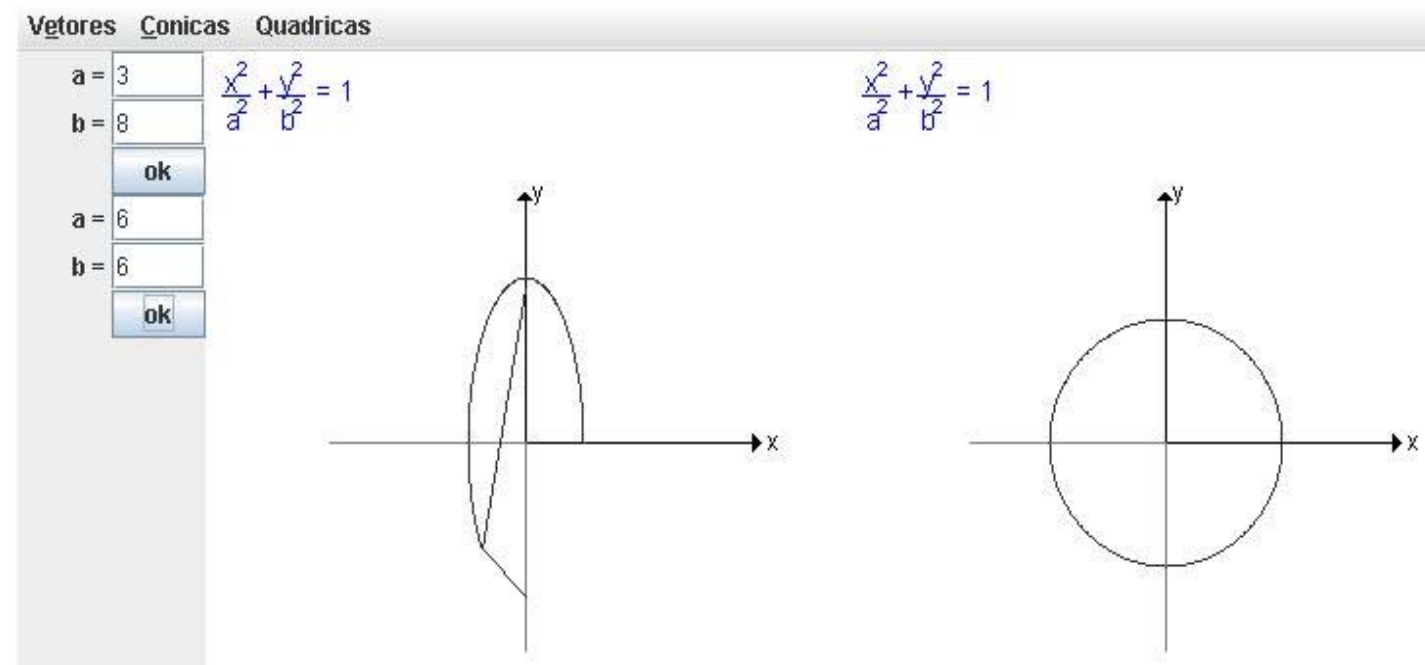

Figura 6 - Tela para Definir e Construir de uma Elipse

A Figura 7 apresenta a construção de duas hipérboles: uma com os focos sobre o eixo x e a outra com os focos sobre o eixo y.

A Figura 8 apresenta uma tela que tem um campo a ser preenchido para a construção de duas parábolas: uma com o foco no eixo x e a outra com o foco no eixo y. Essas cônicas são desenhadas com animação que mostra a definição de uma parábola. 


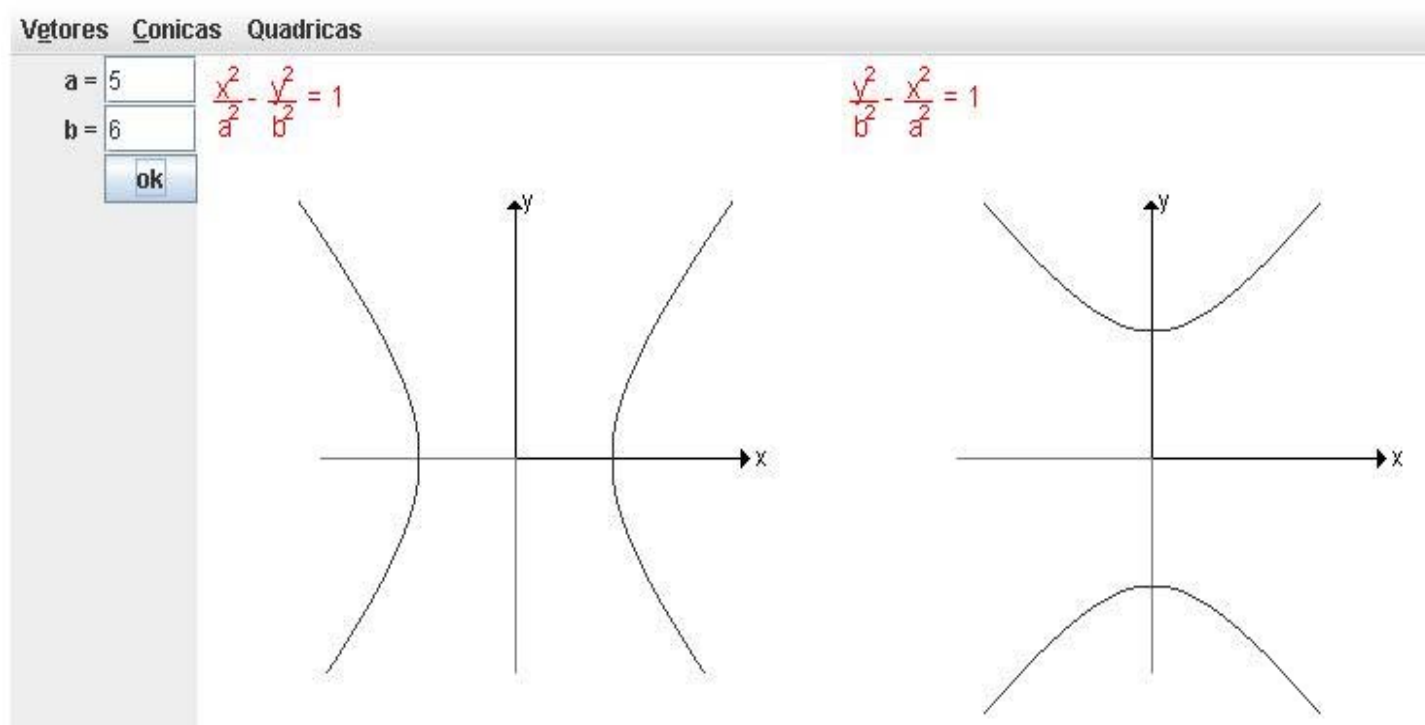

Figura 7 - Tela para Definir e Construir de uma Hipérbole

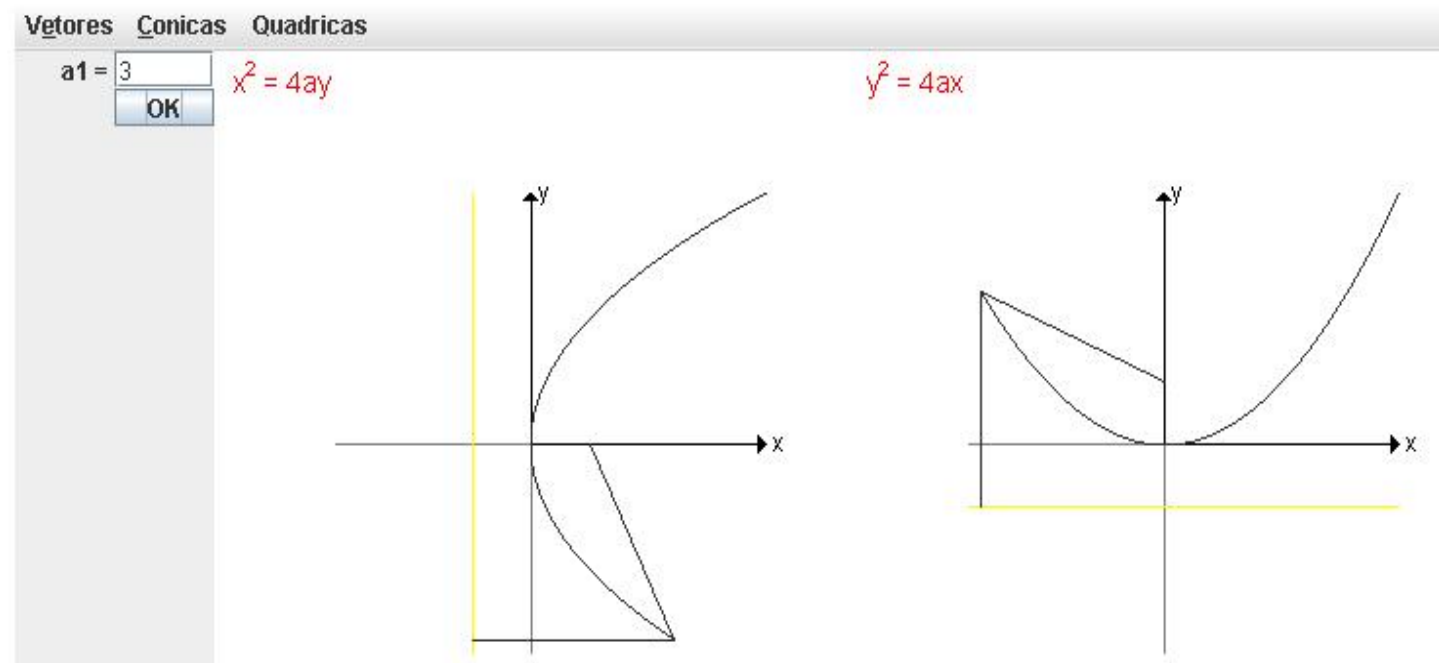

Figura 8 - Mostra Definição e Construção de uma Parábola

A Figura 9 mostra as superfícies que podem ser representadas, quando escolhida a opção Quádricas: i) cilindro; ii) elipsóide; iii) hiperbolóide de 1 e 2 folhas; e iv) parabolóide hiperbólico.

A Figura 10 apresenta uma tela com três opções para construção da superfície cilíndrico parabólico: i) variável z livre; ii) variável x livre; e iii) variável y livre. Quando a variável ausente é $x$, como mostra a figura, têm-se equações da forma f (y, z) $=0$. Neste caso, $\mathrm{f}(\mathrm{y}, \mathrm{z})=0$ e para qualquer valor de $\mathrm{x}$, apresenta-se uma curva no plano yz. 


\begin{tabular}{|l|l|l|}
\hline Vetores Conicas & Quadricas \\
\hline & Eliptico \\
\hline $\begin{array}{l}\text { Elipsoide } \\
\text { Hiperboloide de } 1 \text { folha } \\
\text { Hiperboloide de } 2 \text { folna } \\
\text { Paraboloide Hiperbolico }\end{array}$ & Parabolico \\
\hline
\end{tabular}

Figura 9 - Submenu do Tema Quádricas

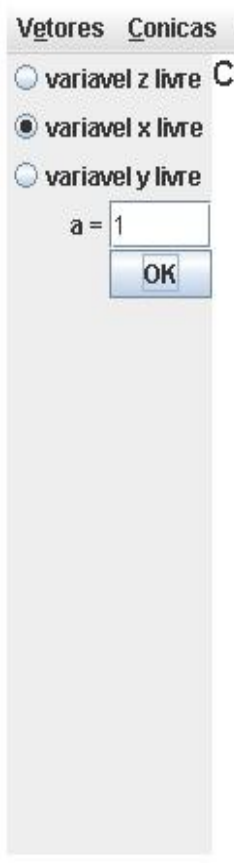

\author{
Cilindro Parabolico
}
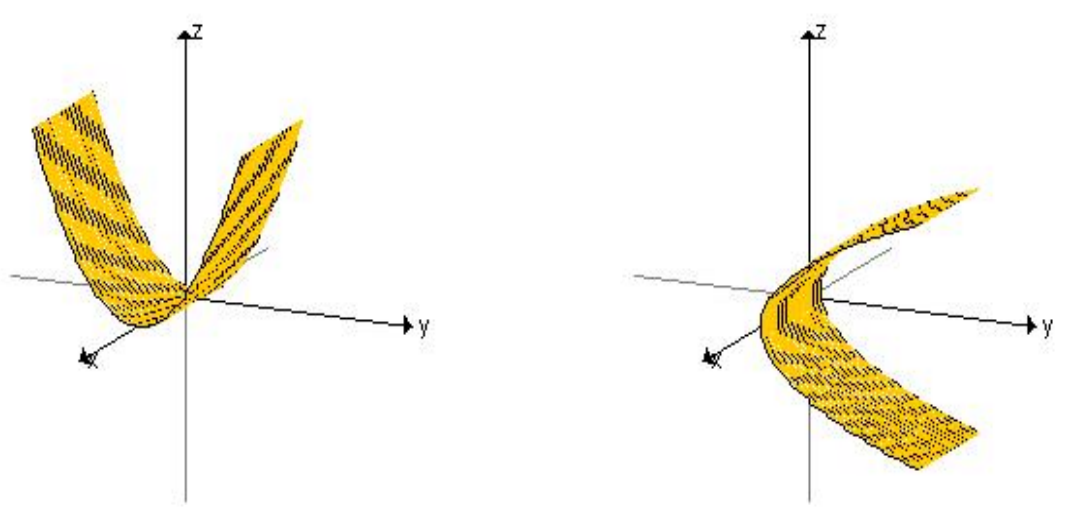

Figura 10 - Cilindro Parabólico

A Tabela 1 apresenta uma comparação entre os ambientes computacionais educacionais para o ensino de geometria analítica e álgebra linear analisados e o TBCGAAL/WEB, segundo as seguintes características: i) software livre; ii) software gratuito; iii) usabilidade (interface intuitiva); iv) código-fonte aberto; e v) Portabilidade. Conforme a percepção e a análise dos autores, pode-se perceber que o TBCGAAL/WEB atende de maneira mais adequada às características comparadas. 
Tabela 1 - Comparação das Ambientes Educacionais e o TBC-GAAL/WEB

\begin{tabular}{|c|c|c|c|c|c|c|}
\cline { 2 - 7 } \multicolumn{1}{c|}{} & WinPlot & C.a.R & Yacas & WinLab & Wingeom & TBC-GAAL/WEB \\
\hline Software livre $^{1}$ & & & $\sqrt{ }$ & & & $\sqrt{ }$ \\
\hline Software gratuito $^{2}$ & $\sqrt{ }$ & $\sqrt{ }$ & $\sqrt{ }$ & $\sqrt{ }$ & $\sqrt{ }$ & $\sqrt{ }$ \\
\hline Usabilidade $^{3}$ & & $\sqrt{ }$ & & $\sqrt{ }$ & $\sqrt{ }$ & $\sqrt{ }$ \\
\hline Código-fonte aberto $^{4}$ & & & $\sqrt{ }$ & & & $\sqrt{ }$ \\
\hline Portabilidade $^{5}$ & & & $\sqrt{ }$ & & & $\sqrt{ }$ \\
\hline
\end{tabular}

\section{Conclusões}

Dentre vários produtos de software educacionais disponíveis para ajudar na compreensão de Geometria Analítica e Álgebra Linear, o aluno que ingressa na universidade não consegue de maneira intuitiva familiarizar com eles. Isso pode ser o resultado do uso inadequado de ótimos ambientes educacionais, os quais possuem uma interface mais elaborada. O TBC-GAAL/WEB tem uma interface bem simples e fácil de usar, de forma que o usuário que ingressa na universidade e interage com ele possa usufruir ao máximo a sua funcionalidade.

Durante a ministração de um mini-curso utilizando o TBC-GAAL/WEB em uma instituição superior de ensino público, pôde-se observar que os participantes o manipularam facilmente. Houve boa aceitação e receptividade dos alunos, uma vez que alguns deles continuaram utilizando nos laboratórios de computadores da sua universidade e no computador de sua casa para complementar e solidificar o conhecimento da disciplina. Assim sendo, o TBC-GAAL/WEB tem contribuído na formação dos alunos e na assimilação do conteúdo apresentado na disciplina de Geometria Analítica e Álgebra Linear.

Conforme as informações apresentadas até o momento, é possível a percepção de uma vasta quantidade de recursos que permitem o desenvolvimento de ferramentas computacionais que auxiliam na educação, enfatizando a linguagem de programação Java e reduzindo a distância entre as novas tecnologias e o seu público alvo.

O uso de produtos de software para o ensino de Geometria Analítica e Álgebra Linear é uma forma inteligente e prática de didática, se for bem planejada, contudo deve ser desenvolvida de forma cuidadosa e estruturada. Isso propicia experiências

\footnotetext{
1 Segundo a definição criada pela Free Software Foundation, é qualquer programa usado, copiado, estudado, modificado e redistribuído com algumas restrições. A liberdade de tais diretrizes é central ao conceito, o qual se opõe ao conceito de software proprietário, mas não ao software que é vendido almejando lucro (software comercial). A maneira usual de distribuição de software livre é anexar a este uma licença de sofwatre livre e tornar o código fonte do programa disponível.

2 Programa de computador gratuito para o público, ou seja, não é preciso pagar licença de uso para utilizá-lo. Por outro lado, a sua comercialização, direta ou incluída em pacotes pagos, não é permitida pelo autor. Pode ser utilizado por período indeterminado (não deixa de funcionar ou perde parcialmente sua funcionalidade, depois de transcorrido certo período). É diferente de software livre ou open source, pois software gratuito não implica que possa ser modificado ou que possa utilizar qualquer parte do programa em um programa próprio.

${ }^{3}$ Termo usado para definir a facilidade com que as pessoas podem empregar uma ferramenta ou objeto a fim de realizar uma tarefa específica e importante. Neste caso, facilidade de usar o software.

${ }^{4}$ Tipo de software cujo código fonte é visível publicamente. O software de código aberto respeita as quatro liberdades definidas pela Free Software Foundation. Porém, não estabelece certas restrições como as contidas na GPL. É advogado pela Iniciativa do Código Aberto (Open Source Initiative).

${ }^{5}$ Programa de computador é a sua capacidade de ser compilado ou executado em diferentes arquiteturas, seja hardware ou software. Também pode ser usado para referir a re-escrita de um código fonte para uma outra linguagem de computador.
} 
inovadoras para docentes e discentes, que se proponham a tal temática, elevando sua qualidade de ensino e capacitando-os no avanço acelerado no campo do conhecimento, através da agilidade de processos didáticos e da participação ativa do educador da área.

É possível observar que produtos tecnológicos bem elaborados facilitam a transmissão de conhecimentos se acompanhados de uma significativa pesquisa no tocante a novas metodologias de ensino. Para isso, devem-se ter, como principal finalidade, contribuições que incentivem o aprimoramento do ensino superior e a formação de profissionais qualificados de acordo com a demanda do mercado e reflita efetivamente na formação de futuros professores. Embora eles tenham passado por tal tipo de experiência no decorrer da graduação, terão grandes interesses em aplicar o processo quando estiverem lecionando.

Analisando tais fatos, fica evidente que a educação poderia e precisa ser reavaliada, uma vez que a formação básica da sociedade se dá através de métodos educacionais. Quanto maior a variedade destes, melhores serão os instrumentos usados na transformação e na evolução do pensamento humano. Cabe à comunidade científica acompanhar esse processo de forma crítica para que esses avanços se mantenham e possam prosseguir visando uma melhoria na qualidade de vida social.

Como trabalhos futuros, pretende-se dar continuidade ao uso do TBCGAAL/WEB durante as aulas, sob o acompanhamento dos professores responsáveis pela disciplina de Geometria Analítica e Álgebra Linear. Essa decisão foi tomada em conseqüência do feedback dos discentes que a utilizaram para estudo e obtiveram sucesso, conforme debates em seminários promovidos e questionários aplicados. Além disso, pretende-se realizar maior aprofundamento das comparações, colocando níveis de comparação e aplicando estatísticas para dar maior fundamentação à percepção e à análise.

\section{REFERÊNCIAS}

Barufi, M. C. B. E-cálculo: Um e-curso de matemática, disponível em http://www.cepa.if.usp.br/e-calculo. Acessado em: 12/12/2006.

Cysneiro, P G. Novas tecnologias no cotidiano da escola. Disponível em: http://www.educacaoonline.pro.br/art_as_novas_tecnologias.asp?f_id_artigo=422. Acessado em: 12/12/2006.

Deitel, H. M., Java - Como Programar. Prentice Hall Brasil. 6 Ed Edição. 2007. 1152 p.

Garcia, I. C.; Rezende, P. J.; Calheiros, F. C. Astral: Um Ambiente para Ensino de Estrutras de Dados através de Animações de Algoritmos. Revista Brasileira de Informática na Educação (RBIE' 1997) n. 01, http://www.inf.ufsc.br/sbcie/revista/nr1/garcia.htm. Ambiente ASTRAL disponível em: www.dcc.unicamp.br/ rezende/ASTRAL/. Acesso: 28 abr 2005.

Grothman, R. C.a.R. - Matemática para Gregos \& Troianos. Disponível em: $<$ http://www.gregosetroianos.mat.br/car/demo.html>. Acessado em: 16/01/2008.

Parris (a), R. Philips Exeter Academy. Mathenatics Department. Disponível em: $<$ http://math.exeter.edu/rparris/winplot.html >. Acessado em: 16/01/2008.

Parris (b), R. Philips Exeter Academy. Mathenatics Department. Disponível em: $<$ http://math.exeter.edu/rparris/peanut/winlabz.exe>. Acessado em: 16/01/2008. 
Parris (c), R. Philips Exeter Academy. Mathenatics Department. Disponível em: $<$ http://math.exeter.edu/rparris/wingeom.html>. Acessado em: 16/01/2008.

Pinkus, A. The Yacas Computer Algebra System. Disponível em: $<$ http://yacas.sourceforge.net/downloads.html>. Acessado em: 16/01/2008.

Ponte, J. P.; Oliveira, H.; Varandas, J. M. O contributo das tecnologias de informação e comunicação para o desenvolvimento do conhecimento e da identidade profissional. Disponível em: http://www.educ.fc.ul.pt/docentes/jponte/docs-pt/03-PonteOli-Var(TIC-Dario).doc. Acessado em: 12/12/2006.

Santos, R. P. dos; Costa, H. A. X. Análise de Metodologias e Ambientes de Ensino para Algoritmos, Estruturas de Dados e Programação aos iniciantes em Computação e Informática. INFOCOMP - Journal of Computer Science, v.5, n.1, p.41-50, Mar. 2006. 